\title{
Linear lichen planus
}

INSERM

\section{Source}

INSERM. (1999). Orphanet: an online rare disease and orphan drug data base. Linear lichen planus. ORPHA:254379

Linear lichen planus (LLP), also referred to as Blaschkoid LP, is a rare type of lichen planus characterized by a linear distribution of lichenoid lesions along the lines of Blaschko, which are embryonic pathways of skin development. 